\title{
A particular dubrheuil melanoma
}

Ouiame EL Jouari ${ }^{*}$, Sara Elloudi ${ }^{1}$, Ghita Senhaji ${ }^{1}$, A Lahlou Mimi ${ }^{3}$, Zakia Douhi ${ }^{1}$, Hanane Baybay ${ }^{1}$, Mouna Rimani ${ }^{2}$ and Fatima Zahra Mernissi $^{1}$

${ }^{1}$ Department of Dermatology University Hospital Hassan II, Fez, Morocco

${ }^{2}$ Hassan center of Anatomopathology, Rabat, Morocco

${ }^{3}$ Department of Radiology University Hospital Hassan II, Fez, Morocco

\section{Commentary}

Dubreuilh melanoma accounts for 4 to $15 \%$ of melanomas. It corresponds to melanoma in situ with a low risk of about $5 \%$ progression to invasive melanoma [1]. It usually presents as a heterogeneous and poorly-defined pigmented macula, sitting electively on the face and neck [2]. We report a very particular case of a young woman with a metastatic ganglionic lentigo maligna.

A 48-year-old female patient who was seen for an asymptomatic pigmented macule in the right malar region of the face, developing for 12 years, gradually increasing in size. The clinical examination revealed a $4 \mathrm{~cm}$ pigmented, well-defined, asymmetrical macule of the right malar, extending to the cantus of the right eye. The lesion was irregularly contoured and infiltrated by location, especially the lower part of the lesion. As well as, an $0.5 \mathrm{~cm}$ erythematous papule in the left side of the lesion, firm in consistency (Figure 1). Presence of multiples painful and fixed cervical lymphadenopathies. Dermoscopy showed an asymmetric heterogeneous pigmentation, rhomboidal structures and follicular obliteration in some places (Figure 2). The histological examination was suggestive of Dubreuilh melanoma (Figures 3 and 4).

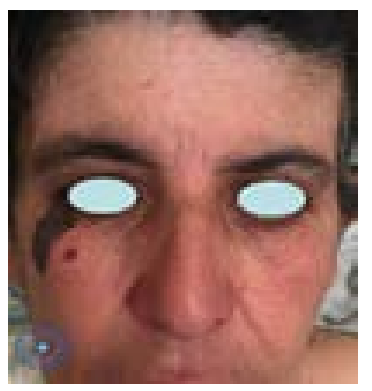

Figure 1. A $4 \mathrm{~cm}$ asymmetrical pigmented macule with irregularly contours
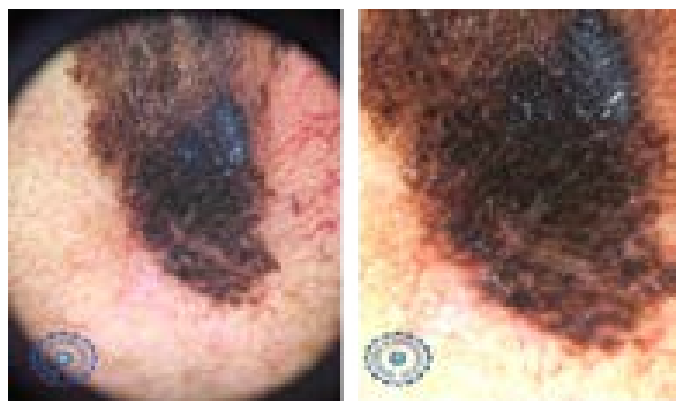

Figure 2. An asymmetric heterogeneous pigmentation with rhomboidal structures and follicular obliteration

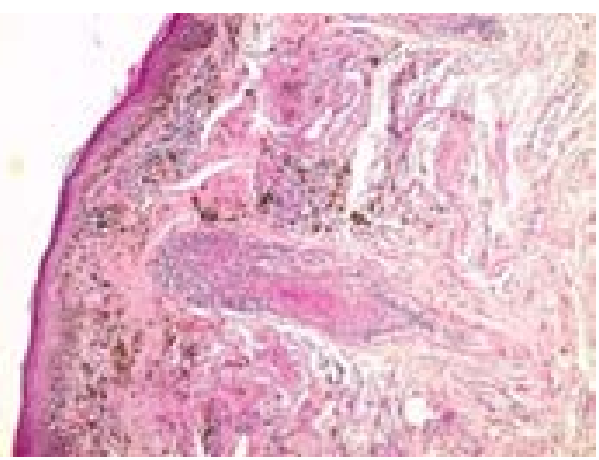

Figure 3. HES stain $\mathrm{G} \times 100 \geq$ lentiginous intraepidermal Melanocyte proliferation + melanophagous in the dermis

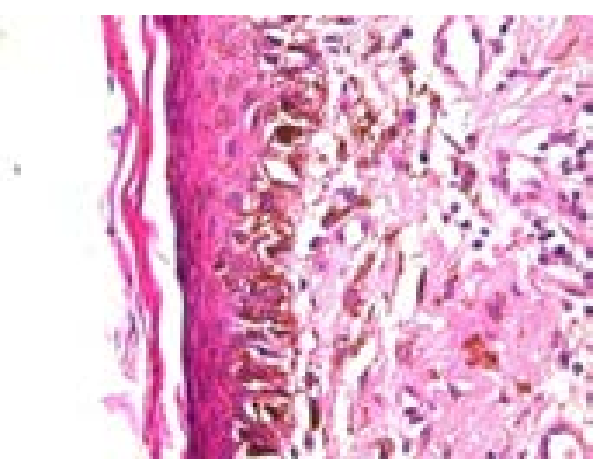

Figure 4. HES stain G x $400 \geq$ Atypical melanocytes

The patient underwent a complete surgical resection with carcinologic margins in the plastic surgery department. The anatompathological examination had objectified a lentigo maligna without any signs of invasion. In our patient, the radiological extension assessment was requested because of the presence of clinically suspected lymphadenopathies, which contrasted with the in situ character of the lentigo maligna. The cerebro-thoraco-abdominopelvic CT revealed several hepatic and pulmonary nodules related to secondary locations with lateral mediastinal and bilateral inguinal lymphadenopathy (Figure 5). A re-reading with new cross-sections of the complete

${ }^{*}$ Correspondence to: Ouiame EL Jouari, Department of Dermatology, University Hospital Hassan II, Fez, Morocco, Tel: +212 5356-19053; E-mail: eljouariouiame88@gmail.com

Received: December 02, 2018; Accepted: December 15, 2018; Published: December 19, 2018 

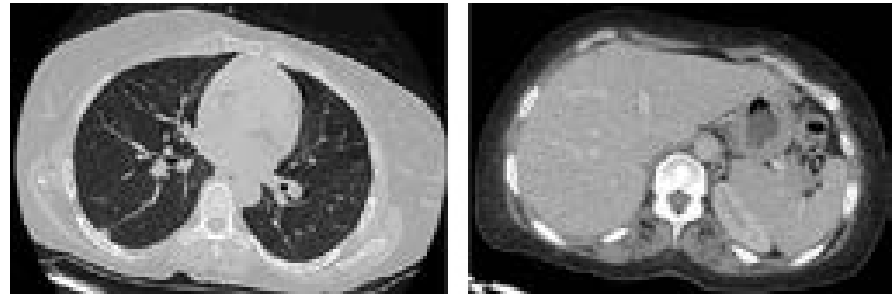

Figure 5. Cerebro-thoraco-abdomino-pelvic CT: Hepatic and pulmonary secondary locations

excision piece was mandatory to explain the metastatic lesions. In our surprise, the anatomopathologist has found a local tumor invasion (Figure 6). The diagnosis of metastatic lentigo maligna melanoma was made. The patient was referred to department of medical oncology for management. A palliative chemotherapy, Dacarabazine, was initiated. The current decline is 12 months with a total regression of the metastatic lesions after 4 cures of chemotherapy.

Jonathan Hutchinson first described the concept of lentigo maligna in 1890. He noted a 'senile freckle' with progressive radial growth that he speculated had an infectious origin. The condition was subsequently further characterized as 'circumscribed precancerous melanosis' by Dubreuilh in 1912 [3]. Lentigo maligna is the most common melanocytic malignancy occurring on sun-exposed skin, usually on the head and neck [4]. An increased incidence of melanoma in younger individuals, with lentigo malgna reported in the fourth decade of life [5]. Risk factors for development of lentigo maligna include the following: a history of sunburns, a history of non-melanoma skin cancers, advanced age, lighter skin types, and tendency to form solar lentigines. Although lentigo maligna occurs on chronically sun-damaged skin, it is thought that intermittent sunburns, rather than cumulative sun exposure, are a risk factor for lentigo maligna [6]. It usually starts as a tan-brown macule or patch, but can have a variegated pigmentation with dark black or even amelanotic features. Lentigo maligna can develop de novo or within a pre-existing solar lentigo [2]. In dermoscopy the dark rhomboidal structures produced by atypical pigment network or dark-brown/ blue- gray ribbonlike structures should be considered as specific for lentigo maligna [7]. Histologically, the first invariable change in LM is a proliferation of atypical melanocytes arranged as solitary units and in small aggregates at the dermoepidermal junction and just above it, within a flat interfollicular epidermis [7]. Fargeas et al. have tried to search for pathological predictive criteria on the biopsy of the invasive nature of the lentigo maligna and they have reported two anatomopathological criteria predictive of lentigo maligna invasion on biopsy: the confluence of the theca and moderate to severe inflammation. Diagnosis and management of lentigo maligna can present several challenges to the clinician and dermatopathologist [1]. Surgical excision remains the 'gold standard' for the treatment of LM [4]. We aim to present this particular case to insight that a rigorous histological examination of the entire specimen is mandatory and the pathological criteria predicting the invasiveness of Dubreuilh's melanoma must be search, to ensure an adequate management. Fortunately our patient had cervical lymphadenopathies which allowed alarming us to realize the radiological assessment of extension and correct the diagnosis to ensure proper management.

\section{References}

1. Hawkey S, Affleck A (2017) Diagnosis and management of lentigo maligna: an observational study comparing 2005 with 2014 data in one institution. Clin Exp Dermatol 42: 320-323. [Crossref]

2. Kasprzak JM, Xu YG (2015) Diagnosis and management of lentigo maligna: a review. Drugs Context 4: 212281. [Crossref]

3. van Ruth S, Toonstra J (2008) Eponyms of Sir Jonathan Hutchinson. Int J Dermatol 47: 754-758. [Crossref]

4. Read T, Noonan C, David M, Wagels M, Foote M, et al. (2016) A systematic review of non-surgical treatments for lentigo maligna. J Eur Acad Dermatol Venereol 30: 748753. [Crossref]

5. Helm MF, Bax MJ, Augenblick DJ, Chung CG, et al. (2017) Melanoma in situ of lentigo maligna type in a young woman. Int J Dermatol 56: 961-962. [Crossref]

6. Gaudy-Marqueste C, Madjlessi N, Guillot B, Avril MF, Grob JJ (2009) Risk factors in elderly people for lentigo maligna compared with other melanomas: a double case control study. Arch Dermatol 145: 418-423. [Crossref]

7. Annessi G, Bono R, Abeni D (2017) Correlation between digital epiluminescence microscopy parameters and histopathological changes in lentigo maligna and solar lentigo: A dermoscopic index for the diagnosis of lentigo maligna. J Am Acad Dermatol 76: 234-243. [Crossref]

Copyright: (C2018 Jouari OEL. This is an open-access article distributed under the terms of the Creative Commons Attribution License, which permits unrestricted use, distribution, and reproduction in any medium, provided the original author and source are credited. 